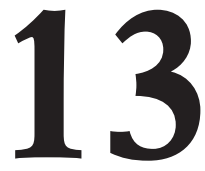

\title{
VARIABLES PERSONALES Y ESCOLARES ASOCIADAS A LA INTELIGENCIA EMOCIONAL ADOLESCENTE
}

\section{(PERSONAL AND SCHOOL VARIABLES ASSOCIATED WITH ADOLESCENT EMOTIONAL INTELLIGENCE)}

José Domínguez Alonso

Begoña Nieto Campos

Universidad de Vigo

Iago Portela Pino

Universidad Isabel I, Burgos

DOI: 10.5944/educXX1.30413

Cómo referenciar este artículo/How to reference this article:

Domínguez Alonso, J., Nieto Campos, B. y Portela Pino, I. (2022). Variables personales y escolares asociadas a la inteligencia emocional adolescente. Educación XX1, 25(1), 335-355. https:// doi.org/10.5944/educXX1.30413

Domínguez Alonso, J., Nieto Campos, B., \& Portela Pino, I. (2022). Personal and school variables associated with adolescent emotional intelligence. Educación XX1, 25(1), 335-355. https:// doi.org/10.5944/educXX1.30413

\section{RESUMEN}

El objetivo de este estudio fue conocer el nivel de inteligencia emocional en el periodo adolescente y analizar la existencia de diferencias significativas que puedan darse en la percepción, comprensión y regulación emocional en función de determinados factores personales y escolares. Participaron 4467 estudiantes de Educación Secundaria Obligatoria (ESO) de 12 a 16 años (M: 13.81; DT: 1.38) de la Comunidad Autónoma de Galicia, a los cuales se administró el instrumento Trait Meta MoodScale (TMMS-24). Los resultados reflejan niveles moderados en la inteligencia emocional de los adolescentes, con una mayor regulación emocional que comprensión y, sobre todo, percepción emocional. Además, los análisis multivariantes muestran diferencias significativas en el género, las 
mujeres presentan mayor percepción emocional y los hombres lo hacen en comprensión y regulación emocional; edad, a mayor edad mejor percepción emocional, mientras que una adolescencia más temprana es indicativa de mejor comprensión y regulación emocional; familia: los adolescentes presentan niveles de regulación emocional más óptimos si viven en familias biparentales; expediente académico, el alumnado que siempre ha aprobado apunta una mejor comprensión y regulación emocional, siendo además dicha comprensión emocional superior en el alumnado repetidor; curso, mejor comprensión y regulación emocional en el primer curso de ESO, mientras la percepción emocional es superior en tercero de ESO; tipo de centro, mayor percepción y regulación emocional en el alumnado que estudia en centros concertados; y ubicación de centro, los núcleos rurales favorecen la percepción y regulación emocional, mientras los urbanos lo hacen en comprensión emocional. En conclusión, se evidencia que la inteligencia emocional está mediatizada por factores personales y escolares en la población adolescente, los cuales deberían servir al profesorado en el establecimiento de estrategias para su incorporación a los centros de enseñanza.

\section{PALABRAS CLAVE}

Inteligencia emocional, enseñanza secundaria, género, familia, expediente académico, centro educativo

\section{ABSTRACT}

The objective of this study was to learn the level of emotional intelligence in the adolescent period and to analyze the existence of significant differences that may occur in perception, understanding and emotional regulation based on certain personal and school factors. 4467 Compulsory Secondary Education students aged 12 to 16 (M: 13.81; DT: 1.38) from the Autonomous Community of Galicia participated, to whom the Trait Meta Mood Scale (TMMS-24) instrument was administered. The results reflect moderate levels of emotional intelligence in adolescents, with greater emotional regulation than understanding and, above all, emotional perception. In addition, multivariate analyses show significant differences in gender, women present greater emotional perception and men do so in understanding and emotional regulation; in age, the older the better emotional perception, while an earlier adolescence is indicative of better understanding and emotional regulation; in family: adolescents have more optimal levels of emotional regulation if they live in two-parent families; in academic record, the students who have always passed point to a better 
understanding and emotional regulation, and this emotional understanding is also higher in repeating students; in course, better understanding and emotional regulation in the first year of ESO, while emotional perception is higher in the third year of ESO; in type of center, greater perception and emotional regulation in students who study in subsidized centers; and downtown location, rural nuclei favor perception and emotional regulation, while urban ones do so in emotional understanding. In conclusion, it is evidenced that emotional intelligence is mediated by personal and school factors in the adolescent population, which should serve teachers in establishing strategies for their incorporation into schools.

\section{KEYWORDS}

Emotional intelligence, secondary education, gender, family, academic record, school

\section{INTRODUCCIÓN}

Durante las dos últimas décadas los sistemas educativos se han caracterizado por un gran número de cambios enfocados, sobre todo, a una transmisión eficiente de conocimientos teóricos, sin atender a las necesidades emocionales del alumnado (García et al., 2018; Peña-Casares y Aguaded-Ramírez, 2019; Ponce y Aguaded, 2016). Esta tendencia debe ser revertida para que la dimensión emocional empiece a tomar relevancia en el ámbito socio-educativo, ya que la inteligencia emocional constituye en la actualidad uno de los mejores predictores de éxito en los entornos académicos y profesionales (Dolev y Leshem, 2017; Menéndez, 2018).

Por tanto, la inteligencia emocional pasa a ser considerada un componente clave en los espacios de enseñanza, con un papel relevante para incrementar el bienestar, las relaciones interpersonales, el rendimiento escolar y, sobre todo, el comportamiento del alumnado (Gómez-Baya et al., 2017; Sánchez-Álvarez et al., 2016). En tal sentido, sería necesario que los centros educativos incorporen en sus currículos contenidos emocionales si buscan mejorar la convivencia y/o el rendimiento académico de sus estudiantes. Este hecho pasa, porque el profesorado no solo haga énfasis en los conocimientos disciplinares y asuma decisiones sobre aspectos relacionados con las competencias emocionales (García et al., 2018).

Teniendo en cuenta la existencia de diferentes modelos teóricos sobre la inteligencia emocional, el presente estudio se ha basado en el Modelo de Salovey y Mayer (1990, p. 189), según el cual la inteligencia emocional hace 
referencia a la capacidad para "monitorizar los sentimientos y emociones de uno mismo y de otros, discriminar entre ellos y utilizar esta información para guiar el propio pensamiento y las propias acciones". Es decir, una habilidad para percibir, valorar y expresar emociones con exactitud, y acceder o generar sentimientos que faciliten el pensamiento (percepción emocional); comprender la información emocional, cómo las emociones se combinan, progresan, y apreciar el significado emocional de los eventos (comprensión emocional); y, estar abierto y modular nuestros sentimientos y los de los demás y promover el crecimiento personal y emocional (regulación emocional). Llevado al ámbito educativo supone "ser capaces de contenerse y refrenar las reacciones impulsivas ante sucesos; tratar con entornos educativos, docentes y temas nuevos; y colaborar con los maestros y otros estudiantes" (Blakemore y Frith, 2007, p. 307).

Así pues, dada la trascendencia que tiene poseer una adecuada inteligencia emocional para los individuos, ha hecho incrementar el número de investigaciones empíricas en esta temática desde dentro y fuera de los contextos escolares, enfocadas fundamentalmente a dificultades que podría acarrear su falta: déficits en los niveles de bienestar y ajuste psicológico del alumnado (Ramos et al., 2007); disminución en la cantidad y calidad de las relaciones interpersonales (Petrides et al., 2006); ansiedad, depresión, enfado y comportamientos disruptivos (Mavroveli et al., 2007); acoso escolar y ciberacoso (Gini y Espelage, 2014; Nixon, 2014) y, sobre todo, disminución en su rendimiento académico (Matthews et al., 2012).

Dentro de esta tendencia prolífica en estudios sobre las emociones, aparecen trabajos que analizan las posibles influencias que pueden tener determinadas variables socio-demográficas para el desarrollo de una mejor inteligencia emocional. Así, unas de las variables más analizadas es el género, no presentando las investigaciones unanimidad en sus conclusiones. Por un lado, emergen estudios que sostienen diferencias significativas en la inteligencia emocional según el género, indicando mayores niveles en el género femenino que en el masculino (Azpiazu et al., 2015; Pulido y Herrera, 2016). Por otro, trabajos como el realizado por Ruiz y Carranza (2018) sostienen que las mujeres y los hombres no presentaron diferencias en la puntuación total de la inteligencia emocional. Pero también existen investigaciones que señalan mayor claridad y atención a sus emociones en el género femenino, mientras el masculino lo hace en el manejo de sus emociones (Saucedo et al., 2019).

Otra de las variables estudiadas es la edad. Son numerosos los estudios que indican una mayor inteligencia emocional a medida que se incrementa la edad (Pulido y Herrera, 2018). De igual manera, el estatus socioeconómico y cultural también puede determinar la inteligencia emocional, mostrando 
mayor competencia emocional los individuos que pertenecen a estatus superiores (Pulido y Herrera, 2016).

En consecuencia, las nuevas exigencias que deben afrontar los centros educativos en cuanto a satisfacer necesidades de índole socioemocional del alumnado, constituyen un motivo más para la inclusión de competencias emocionales entre las tareas de los agentes educativos. De hecho, debe ser enfocada como un proceso educativo, continuo y permanente en los centros de enseñanza, constituyendo un elemento esencial en el desarrollo integral del individuo, con el propósito de capacitarlo para afrontar mejor los retos que se planteen en su vida cotidiana (Bisquerra, 2012). Así, Alonso-Ferres et al. (2018) indican que "la inteligencia emocional es un factor relevante en la vida de cualquier ciudadano, es por ello que resulta fundamental trabajarla en los centros educativos" (p. 156).

En definitiva, este trabajo de investigación examina un doble objetivo: conocer los promedios de inteligencia emocional en el periodo adolescente y analizar si las variables personales (género, edad y familia) y escolares (curso escolar, expediente académico, tipo y ubicación del centro de enseñanza) generan diferencias estadísticamente significativas en las dimensiones (percepción, comprensión y regulación) de la inteligencia emocional. Asimismo, se han establecido como hipótesis de investigación: $\mathrm{H}_{1}$. "Se infieren promedios moderados en la percepción, comprensión y regulación emocional durante la adolescencia” y $\mathrm{H}_{2}$. "Se predice que género, edad, estructura familiar, curso escolar, expediente académico, ubicación y tipo de centro, presenten diferencias significativas en la percepción, comprensión y regulación emocional durante la adolescencia del individuo”.

\section{MÉTODO}

\section{Participantes}

Se ha utilizado un muestreo estratificado donde la primera unidad fue la titularidad de los centros y la etapa de ESO. La muestra quedó formada por un total de 4467 estudiantes de los cuales el $50.26 \%$ son mujeres y el $49.74 \%$ son hombres. La media de edad expresada en años es de $13.81 \pm 1.38$ (20.39\% de 12 años, 23.48\% de 13 años, 23.64\% de 14 años, 21.60\% de 15 años y $10.89 \%$ de 16 años). Asimismo, el $81.04 \%$ vive en familias biparentales frente a un $18.96 \%$ que lo hace en familias monoparentales. De igual manera, un 59.14\% del alumnado presenta un expediente académico de aprobado, frente al $16.08 \%$ que tiene alguna materia suspensa y el $24.78 \%$ que ha tenido que repetir algún curso. 
Si se tiene en cuenta el centro educativo, el 50.93\% del alumnado estudia en centros ubicados en entornos rurales y el $49.07 \%$ lo hace en centros emplazados en zonas urbanas. Además, el 78.06\% está matriculado en centros de titularidad pública, frente al $21.94 \%$ que lo hace en centros concertados. Finalmente, indicar que el $26.75 \%$ del alumnado se encuentra matriculado en $1^{\circ}$ de ESO, el $26.93 \%$ en $2^{\circ}$ de ESO, el $23.89 \%$ en $3^{\circ}$ de ESO y el $22.43 \%$ lo hace en $4^{\circ}$ de ESO.

\section{Instrumentos}

\section{Cuestionario socio-escolar}

Con el propósito de recabar información relevante se han incluido siete variables socio-escolares para recopilar datos personales (género, edad, estructura familiar) y escolares (curso escolar, expediente académico, ubicación y tipo de centro) con la intención de obtener información de la muestra y analizar la existencia de diferencias significativas en función de las mismas.

\section{Trait Meta-Mood Scale-24 (TMMS-24)}

El Trait Meta Mood Scale-24 (traducido y adaptado al castellano por Fernández-Berrocal et al., 2004) es una prueba de autoinforme de la inteligencia emocional percibida compuesta por 24 ítems en una escala Likert de cinco puntos $(1=$ Totalmente en desacuerdo, 5 = Totalmente de acuerdo) repartidos en tres dimensiones: percepción (nivel de creencia sobre la focalización emocional), comprensión (percepción subjetiva sobre las propias emociones) y regulación emocional (creencia de poder interrumpir y regular los estados emocionales negativos y potenciar los positivos).

Desde esta perspectiva, las medidas de autoinforme o pruebas de habilidad son las más utilizadas en la evaluación de la inteligencia emocional. Según González et al. (2011), las primeras posibilitan valoraciones subjetivas de las competencias emocionales, mientras que las segundas examinan dichas habilidades a través de ejercicios. Así pues, teniendo en cuenta la naturaleza y los objetivos de la investigación (Antonio, 2019; Antonio et al., 2017) se ha adoptado por el empleo de la escala Trait Meta-MoodScale (TMMS-24) frente a otro tipo de medidas, fundamentalmente por ser la más utilizada, su fácil aplicación, sus excelentes propiedades psicométricas, las dimensiones que evalúa, los antecedentes disponibles de adaptaciones y validaciones en poblaciones de habla hispana, y sobre todo, por los numerosos estudios actuales sobre inteligencia emocional que se han decantado por el uso de esta escala de medida (Carrasco, 2017; Márquez, 2016; Ocaña et al., 2019; Taramuel y Zapata, 2017). 
Se han realizado estudios de validez y confiabilidad dividiendo la muestra obtenida en dos mitades aleatoriamente. Con la primera muestra (2233 alumnos) se ha llevado a cabo un Análisis Factorial de Componentes Principales, alcanzando valores adecuados para el índice KMO (.926) como en el test de esfericidad de Barlett $\left(\chi_{276}^{2}=43916.896 ; p<.001\right)$. La estructura tridimensional resultante (igual a la original del cuestionario) estaba conformada por tres factores (percepción, comprensión y regulación) que conjuntamente explican un $51.88 \%$ de la varianza. A continuación, con la segunda muestra (2234 alumnos) se procedió a la realización del Análisis Factorial Confirmatorio, presentando un ajuste aceptable de los datos $\left(\chi^{2}{ }_{(249)}\right.$ $=5319.338, p<.001$, RMSEA $=.068$, intervalo de confianza 90\% (CIs) [.066 -.069], SRMR $=.073$, GFI $=.895, \mathrm{NNFI}=.889$ y CFI $=.891$ ). Finalmente, con la totalidad de la muestra se ha comprobado la fiabilidad del instrumento medida a través del Alpha de Cronbach, obteniendo un valor de.89 para los 24 ítems (percepción:.87; comprensión:.86; regulación:.84).

\section{Procedimiento}

En primer lugar, se ha establecido una reunión con los equipos directivos de los centros educativos para solicitar su libre colaboración en el estudio. Al respecto, los participantes en la misma fueron informados del propósito del trabajo, confidencialidad y anonimato. Se ha requerido el consentimiento informado de sus familias o tutores. Todo ello bajo las normas éticas de la Declaración de Helsinki 1961 y aprobado por la Comisión de Ética de la Universidad de Vigo (España).

Para la codificación y análisis de los datos se empleó el programa estadístico SPSS versión 23 (IBM Corp., 2012). Se ha realizado en primer lugar, el análisis de la validez y confiabilidad del cuestionario para esta muestra (apartado instrumentos). En segundo lugar, se ha llevado a cabo un análisis descriptivo (medias y desviaciones típicas). Finalmente, se utiliza el análisis multivariante a través de MANOVA para conocer la existencia de diferencias significativas a nivel estadístico de la percepción, comprensión y regulación emocional en función de las variables personales y escolares.

\section{RESULTADOS}

\section{Análisis descriptivo de Inteligencia Emocional}

Atendiendo a los resultados alcanzados (Tabla 1), se observa que el factor de inteligencia emocional más desarrollado en el alumnado de ESO es: la regulación emocional $(M=3.33 ; D T=1.32)$, señalando que tienen 
mucha energía cuando se sienten felices o manifiestan su preocupación por tener un buen estado de ánimo; seguido de la comprensión $(M=3.08$; $D T=1.25)$, indicando que saben siempre cómo se sienten o se dan cuenta de sus sentimientos en diferentes situaciones; y finalmente, la percepción emocional $(M=2.98 ; D T=1.24)$ prestando mucha atención o preocupación por sus sentimientos.

\section{Tabla 1}

Descriptivos de los items por factores que conforman la inteligencia emocional

\begin{tabular}{|c|c|c|}
\hline PERCEPCIÓN (M: 2.98; DT: 1.24) & $M$ & $D T$ \\
\hline Presto mucha atención a los sentimientos & 3.47 & 1.17 \\
\hline Normalmente me preocupo mucho por lo que siento & 3.33 & 1.20 \\
\hline $\begin{array}{l}\text { Pienso que merece la pena prestar atención a mis emociones y } \\
\text { estado de ánimo }\end{array}$ & 3.30 & 1.25 \\
\hline Normalmente me preocupo mucho por lo que siento & 2.90 & 1.25 \\
\hline Presto mucha atención a cómo me siento & 2.89 & 1.23 \\
\hline A menudo pienso en mis sentimientos & 2.82 & 1.24 \\
\hline Dejo que mis sentimientos afecten a mis pensamientos & 2.57 & 1.33 \\
\hline Pienso en mi estado de ánimo constantemente & 2.56 & 1.28 \\
\hline COMPRENSIÓN $(M: 3.08 ; D T: 1.25)$ & $M$ & $D T$ \\
\hline Casi siempre sé cómo me siento & 3.24 & 1.29 \\
\hline $\begin{array}{l}\text { A menudo me doy cuenta de mis sentimientos en diferentes si- } \\
\text { tuaciones }\end{array}$ & 3.24 & 1.18 \\
\hline Normalmente conozco mis sentimientos sobre las personas & 3.22 & 1.22 \\
\hline Tengo claros mis sentimientos & 3.20 & 1.38 \\
\hline Puedo llegar a comprender mis sentimientos & 3.18 & 1.22 \\
\hline A veces puedo decir cuáles son mis emociones & 2.95 & 1.20 \\
\hline Siempre puedo decir cómo me siento & 2.83 & 1.31 \\
\hline Frecuentemente puedo definir mis sentimientos & 2.80 & 1.25 \\
\hline REGULACIÓN $(M: 3.33 ; D T: 1.32)$ & $M$ & $D T$ \\
\hline Tengo mucha energía cuando me siento feliz & 4.31 & 1.07 \\
\hline Me preocupo por tener un buen estado de ánimo & 3.43 & 1.27 \\
\hline Aunque me siento mal, procuro pensar en cosas agradables & 3.29 & 1.37 \\
\hline $\begin{array}{l}\text { Aunque a veces me siento triste, pienso en todos los placeres } \\
\text { de la vida }\end{array}$ & 3.24 & 1.38 \\
\hline Intento tener pensamientos positivos, aunque me sienta mal & 3.22 & 1.36 \\
\hline
\end{tabular}




\begin{tabular}{lcc}
\hline PERCEPCIÓN ( $M:$ : 2.98; DT: 1.24$)$ & $\boldsymbol{M}$ & $\boldsymbol{D T}$ \\
\hline Cuando estoy enfadado intento cambiar mi estado de ánimo & 3.16 & 1.38 \\
\hline $\begin{array}{l}\text { Si doy demasiadas vueltas a las cosas, complicándolas, trato de } \\
\text { calmarme }\end{array}$ & 3.10 & 1.34 \\
\hline Cuando estoy triste, pienso en todos los placeres de la vida & 2.85 & 1.41 \\
\hline
\end{tabular}

Nota. M: media; DT: desviación típica

Inteligencia Emocional: diferencias en función de las variables personales

Teniendo en cuenta las variables personales, medias y desviaciones típicas obtenidas por los distintos grupos (femenino y masculino en el género; estructurada y desestructurada en el tipo de familia; 12, 13, 14, 15 y 16 años según la edad) en las puntuaciones de las distintas subescalas que conforman la Inteligencia Emocional (TMMS-24): Percepción Emocional (PE), Comprensión Emocional (CE), y Regulación Emocional (RE), se encuentran en la Tabla 2.

\section{Tabla 2}

Medias y Desviaciones Típicas de las subescalas del cuestionario TMMS-24(PE, CE, $R E)$ en función del género, tipo de familia y edad de los adolescentes

\begin{tabular}{|c|c|c|c|c|c|c|}
\hline \multicolumn{2}{|c|}{ GENERO } & \multicolumn{2}{|c|}{ Femenino $(N=2245)$} & \multicolumn{2}{|c|}{ Masculino $(N=2222)$} & Total \\
\hline \multicolumn{2}{|c|}{$\mathrm{PE}$} & \multicolumn{2}{|c|}{$24.63(7.15)$} & \multicolumn{2}{|c|}{$23.04(7.29)$} & $23.84(7.27)$ \\
\hline \multicolumn{2}{|c|}{$\mathrm{CE}$} & \multicolumn{2}{|c|}{$24.02(7.18)$} & \multicolumn{2}{|c|}{$25.28(7.02)$} & $24.65(7.13)$ \\
\hline \multicolumn{2}{|c|}{$\mathrm{RE}$} & \multicolumn{2}{|c|}{$26.21(7.27)$} & \multicolumn{2}{|c|}{$26.99(7.16)$} & $26.60(7.23)$ \\
\hline \multicolumn{2}{|c|}{$\begin{array}{l}\text { ESTRUCTURA } \\
\text { FAMILIAR } \\
\end{array}$} & \multicolumn{2}{|c|}{ Estructurada $(N=3620)$} & \multicolumn{2}{|c|}{$\begin{array}{c}\text { Desestructurada } \\
(N=847)\end{array}$} & Total \\
\hline \multirow{3}{*}{\multicolumn{2}{|c|}{$\begin{array}{l}\mathrm{PE} \\
\mathrm{CE} \\
\mathrm{RE}\end{array}$}} & \multirow{3}{*}{\multicolumn{2}{|c|}{$\begin{array}{l}23.84(7.14) \\
24.67(7.03) \\
26.76(7.12)\end{array}$}} & \multirow{3}{*}{\multicolumn{2}{|c|}{$\begin{array}{l}23.82(7.77) \\
24.56(7.52) \\
25.91(7.62)\end{array}$}} & $23.84(7.27)$ \\
\hline & & & & & & $24.65(7.13)$ \\
\hline & & & & & & $26.60(7.23)$ \\
\hline EDAD & $\begin{array}{l}12 \text { años } \\
(N=911)\end{array}$ & $\begin{array}{c}13 \text { años } \\
(N=1049)\end{array}$ & $\begin{array}{c}14 \text { años } \\
(N=1056)\end{array}$ & $\begin{array}{l}15 \text { años } \\
(N=965)\end{array}$ & $\begin{array}{l}16 \text { años } \\
(N=486)\end{array}$ & Total \\
\hline $\mathrm{PE}$ & $23.72(7.03)$ & $23.58(7.22)$ & $23.53(7.26)$ & $24.38(7.37)$ & $24.21(7.57)$ & $23.84(7.27)$ \\
\hline $\mathrm{CE}$ & $25.48(6.84)$ & $24.51(7.29)$ & $24.37(7.05)$ & $24.28(7.05)$ & $24.72(7.50)$ & $24.65(7.13)$ \\
\hline $\mathrm{RE}$ & $28.31(6.65)$ & $27.02(7.12)$ & $26.07(7.28)$ & $25.57(7.19)$ & $25.67(7.82)$ & $26.60(7.23)$ \\
\hline
\end{tabular}

Nota . PE = Percepción Emocional; $\mathrm{CE}=$ Comprensión Emocional; RE = Regulación Emocional

Los resultados del MANOVA revelaron que existían diferencias significativas entre los individuos de género femenino y masculino en inteligencia emocional en las puntuaciones de las distintas subescalas del TMMS-24 (PE, CE, RE) [Lambda del Wilks $=.97, F(3,4463)=45.97, p<.001$, $\eta^{2}=.030$, potencia $\left.=1\right]$; los adolescentes que viven en familias estructuradas y desestructuradas en las puntuaciones de las subescalas PE, CE y RE [Lambda del Wilks $=.99, F(3,4463)=3.71, p<.05, \eta_{\mathrm{p}}^{2}=.002$, potencia $\left.=.81\right]$; 
y la edad (12, 13, 14, 15 y 16 años) en las puntuaciones de las distintas subescalas del TMMS-24 (PE, CE, RE) [Lambda del Wilks =.97, $F(4,4462)$ $=10.09, p<.001, \eta^{2}{ }_{p}=.009$, potencia $\left.=1\right]$.

Los análisis univariados (Tabla 3) también mostraron diferencias significativas en género, estructura familiar y edad con las diferentes subescalas del TMMS-24 (PE, CE, RE). Las diferencias de género fueron significativas para la percepción emocional $(p=.000)$, comprensión emocional $(p=.000)$ y regulación emocional $(p=.000)$. En consecuencia, los individuos de género femenino tenían mejor percepción emocional ( $M$ $=24.63, S D=7.15)$ que los de género masculino $(M=23.04, S D=7.29)$, mientras que los individuos de género masculino tenían mejor comprensión emocional $(M=25.28, S D=7.02)$ y regulación emocional $(M=26.99, S D=$ 7.16) que los de género femenino (CE: $M=24.02, S D=7.18$; RE: $M=26.21$, $S D=7.27$.

Atendiendo a la estructura familiar, no resultó significativa en la percepción emocional ( $p=.939)$ y la comprensión emocional $(p=.657)$, pero sí fue significativa en la regulación emocional $(p=.004)$. Así, los individuos que viven en familias estructuradas $(M=26.76, S D=7.12)$ mostraban mayor regulación emocional que los de familias desestructuradas $(M=$ 25.91, $S D=7.62$ ).

Finalmente, las diferencias de edad fueron significativas para la percepción emocional $(p=.037)$, comprensión emocional $(p=.002)$ y regulación emocional ( $p=.000$ ). El análisis post hoc de Tukey reveló que los individuos con mayor edad (15 años) tenían mejor percepción emocional ( $M$ $=24.38, S D=7.37$ ) que los individuos de menor edad (13 años: $M=23.58$, $S D=7.22$, y 14 años: $M=23.53, S D=.7 .26$ ); los individuos de menor edad (12 años: $M=25.48, S D=6.84$ ) tenían mejor comprensión emocional que los de mayor edad (14 años: $M=24.37, S D=7.05$, y 15 años: $M=24.28, S D$ = 7.05); y los individuos de menor edad (12 años: $M=28.31, S D=6.65$, y 13 años: $M=27.02, S D=7.12)$ tenían mejor regulación emocional que los de mayor edad (14 años: $M=26.07, S D=7.28,15$ años: $M=25.57, S D=7.19$, y 16 años: $M=25.67, S D=7.82$ ). 


\section{Tabla 3}

Análisis Multivariado de Varianza (MANOVA). Variables independientes: género, familia y edad. Variables dependientes: subescalas de la inteligencia emocional (TMMS-24)

\begin{tabular}{cccccc}
\hline $\begin{array}{c}\text { VARIABLES } \\
\text { INDEPENDIENTES }\end{array}$ & $\begin{array}{c}\text { SUBESCALAS } \\
\text { IE }\end{array}$ & $\boldsymbol{F}$ & $\boldsymbol{p}$ & $\eta_{\mathbf{p}}{ }^{2}$ & Potencia \\
\hline Género & $\mathrm{PE}$ & 53.93 & .000 & .012 & 1 \\
& $\mathrm{CE}$ & 34.82 & .000 & .008 & 1.95 \\
& $\mathrm{RE}$ & 13.04 & .000 & .003 & \\
\hline \multirow{2}{*}{ Familia } & $\mathrm{PE}$ & .006 & .939 & .000 & .05 .07 .87 \\
& $\mathrm{CE}$ & .176 & .657 & .000 & \\
& $\mathrm{RE}$ & 9.39 & .004 & .002 & \\
\hline Edad & $\mathrm{PE}$ & 2.55 & .037 & .002 & .73 .93 \\
& $\mathrm{CE}$ & 4.28 & .002 & .004 & 1 \\
& $\mathrm{RE}$ & 22.19 & .000 & .020 & \\
\hline
\end{tabular}

Nota IE = Inteligencia Emocional; PE = Percepción Emocional; CE = Comprensión Emocional; $\mathrm{RE}=$ Regulación Emocional

Inteligencia Emocional: diferencias en función de las variables escolares

En las variables escolares, medias y desviaciones típicas obtenidas por los distintos grupos (Curso Escolar: $1^{\circ} \mathrm{ESO}, 2^{\circ} \mathrm{ESO}, 3^{\circ} \mathrm{ESO}, 4^{\circ} \mathrm{ESO}$; Expediente Académico: aprobar, suspender, repetir; Tipo de centro: público y concertado; y Ubicación del centro: rural y urbano) en las puntuaciones de las distintas subescalas que conforman la Inteligencia Emocional (TMMS-24): Percepción Emocional (PE), Comprensión Emocional (CE), y Regulación Emocional (RE), se encuentran en la Tabla 4.

Los resultados del MANOVA indicaron que existían diferencias significativas entre los individuos que cursaban $1^{\circ}, 2^{\circ}, 3^{\circ}$ o $4^{\circ}$ de ESO en las puntuaciones de las subescalas del TMMS-24: Percepción Emocional (PE), Comprensión Emocional (CE), y Regulación Emocional (RE) [Lambda del Wilks $=.97, F(3,4463)=13.20, p<.001, \eta^{2}{ }_{p}=.009$, potencia $\left.=1\right]$; entre el expediente académico (aprobar, suspender y repetir) en las puntuaciones de las subescalas comprensión y regulación emocional [Lambda del Wilks =.99, $F(3,4463)=9.71, p<.001, \dot{\eta}_{p}^{2}=.006$, potencia $\left.=1\right]$; entre el tipo de centro (público y concertado) en las puntuaciones de las subescalas percepción y regulación emocional [Lambda del Wilks $=.99, F(3,4463)=6.91, p<.001$, $\eta_{\mathrm{p}}^{2}=.005$, potencia $=.98$ ]; y entre la ubicación del centro (rural y urbana) en las puntuaciones de las subescalas percepción, comprensión y regulación emocional [Lambda del Wilks $=.99, F(3,4463)=10.26, p<.001, \eta_{\mathrm{p}}^{2}=.007$, potencia $=.99]$. 


\section{Tabla 4}

Medias y Desviaciones Típicas de las subescalas del cuestionario TMMS-24(PE, CE, $R E$ ) en función del curso escolar, expediente académico, tipo y ubicación del centro

\begin{tabular}{|c|c|c|c|c|c|}
\hline $\begin{array}{c}\text { CURSO } \\
\text { ESCOLAR }\end{array}$ & $\begin{array}{c}1^{o} E S O \\
(N=1195)\end{array}$ & $\begin{array}{c}2^{o} E S O \\
(N=1203)\end{array}$ & $\begin{array}{c}3^{\circ} \mathrm{ESO} \\
(N=1067)\end{array}$ & $\begin{array}{c}4^{\circ} E S O \\
(N=1002)\end{array}$ & Total \\
\hline $\mathrm{PE}$ & $23.69(7.13)$ & $23.38(7.29)$ & $24.26(7.34)$ & $24.12(7.29)$ & $23.84(7.27)$ \\
\hline $\mathrm{CE}$ & $25.49(6.98)$ & $24.16(7.33)$ & $24.63(7.21)$ & $24.24(6.89)$ & $24.65(7.13)$ \\
\hline $\mathrm{RE}$ & $28.11(6.88)$ & $26.31(7.35)$ & $26.46(7.24)$ & $25.28(7.15)$ & $26.60(7.23)$ \\
\hline $\begin{array}{c}\text { EXP. } \\
\text { ACADÉMICO }\end{array}$ & \multicolumn{2}{|c|}{$\begin{array}{l}\text { Aprobar } \\
(N=2642)\end{array}$} & $\begin{array}{c}\text { Suspender } \\
(N=718)\end{array}$ & $\begin{array}{c}\text { Repetir } \\
(N=1107)\end{array}$ & Total \\
\hline $\mathrm{PE}$ & \multicolumn{2}{|c|}{$23.98(7.14)$} & $23.30(7.22)$ & $23.87(7.58)$ & $23.84(7.27)$ \\
\hline $\mathrm{CE}$ & \multicolumn{2}{|c|}{$25.06(6.93)$} & $23.37(7.20)$ & $24.50(7.43)$ & $24.65(7.13)$ \\
\hline RE & \multicolumn{2}{|c|}{$27.16(6.88)$} & $25.73(7.31)$ & $25.80(7.84)$ & $26.60(7.23)$ \\
\hline $\begin{array}{l}\text { TIPO DE } \\
\text { CENTRO }\end{array}$ & \multicolumn{2}{|c|}{ Público $(N=3487)$} & \multicolumn{2}{|c|}{ Concertado $(N=980)$} & Total \\
\hline $\mathrm{PE}$ & \multicolumn{2}{|c|}{$23.59(7.28)$} & \multicolumn{2}{|c|}{$24.71(7.13)$} & $23.84(7.27)$ \\
\hline $\mathrm{CE}$ & \multicolumn{2}{|c|}{$24.57(7.14)$} & \multicolumn{2}{|c|}{$24.94(7.06)$} & $24.65(7.13)$ \\
\hline RE & \multicolumn{2}{|c|}{$26.45(7.25)$} & \multicolumn{2}{|c|}{$27.12(7.13)$} & $26.60(7.23)$ \\
\hline $\begin{array}{l}\text { UBICACIÓN } \\
\text { DEL CENTRO }\end{array}$ & \multicolumn{2}{|c|}{ Rural $(N=2275)$} & \multicolumn{2}{|c|}{ Urbano $(N=2192)$} & Total \\
\hline $\mathrm{PE}$ & \multicolumn{2}{|c|}{$24.18(7.33)$} & \multicolumn{2}{|c|}{$23.48(7.18)$} & $23.84(7.27)$ \\
\hline $\mathrm{CE}$ & \multicolumn{2}{|c|}{$24.43(7.04)$} & \multicolumn{2}{|c|}{$24.87(7.21)$} & $24.65(7.13)$ \\
\hline $\mathrm{RE}$ & \multicolumn{2}{|c|}{$26.84(7.22)$} & \multicolumn{2}{|c|}{$26.35(7.23)$} & $26.60(7.23)$ \\
\hline
\end{tabular}

Nota. PE = Percepción Emocional; $\mathrm{CE}=$ Comprensión Emocional; $\mathrm{RE}=$ Regulación Emocional

Los análisis univariados (Tabla 5) mostraron que el curso escolar ha sido significativo para la percepción emocional $(p=.016)$, comprensión emocional $(p=.000)$ y regulación emocional $(p=.000)$. El análisis post hoc de Tukey evidenció que los individuos que cursaban $3^{\circ}$ ESO $(M=24.26, S D=7.34)$ tenían una mejor percepción emocional que los matriculados en $4^{\circ}$ ESO $(M=$ $24.12, S D=7.29)$; los que cursaban $1^{\circ} \mathrm{ESO}(M=25.49, S D=6.98)$ tenían una mejor comprensión emocional que los matriculados en cursos superiores $\left(2^{\circ}\right.$ ESO: $M=24.16, S D=7.33,3^{\circ}$ ESO: $M=24.63, S D=7.21, y 4^{\circ}$ ESO $: M=24.24$, $S D=6.89$ ); y los matriculados en cursos inferiores ( $1^{\circ}$ ESO: $M=28.11, S D=$ 6.88, $2^{\circ}$ ESO: $M=26.31, S D=7.35$, y3 ${ }^{\circ}$ ESO: $\left.M=26.46, S D=7.24\right)$ tenían una mejor regulación emocional que los que cursan $4^{\circ} \mathrm{ESO}(M=25.28, S D=7.15)$.

Atendiendo al expediente académico, no fue significativo para la percepción emocional $(p=.087)$, pero sí ha resultado significativo en la comprensión emocional $(p=.000)$ y la regulación emocional $(p=.000)$. El análisis post hoc de Tukey ha revelado que los individuos que siempre han aprobado $(M=25.06, S D=6.93)$ o han repetido algún curso $(M=24.50$, $S D=7.43$ ) han tenido significativamente mayor comprensión emocional que los que han suspendido alguna materia en su trayectoria académica 
$(M=23.37, S D=7.20)$. Asimismo, los individuos que han aprobado $(M=$ $27.16, S D=6.88)$ han tenido mejor regulación emocional que los que han suspendido alguna materia $(M=25.73, S D=7.31)$ o han repetido algún curso $(M=25.80, S D=7.84)$.

De igual manera, el tipo de centro no ha resultado significativo en la comprensión emocional $(p=.150)$, pero sí ha sido significativo para la percepción emocional $(p=.000)$ y la regulación emocional $(p=.010)$. Así, los individuos que estudian en centros concertados (PE: $M=24.71, S D=7.13$; RE: $M=27.12, S D=7.13)$ tenían mejor percepción y regulación emocional que los estudiantes de centros de titularidad pública (PE: $M=23.59, S D=$ 7.28; RE: $M=26.45, S D=7.25$ ).

Finalmente, las diferencias en la ubicación del centro han sido significativas para la percepción emocional $(p=.001)$, comprensión emocional $(p=.042)$ y regulación emocional $(p=.022)$. En consecuencia, los individuos que cursan estudios de ESO en centros rurales tenían mejor percepción ( $M$ $=24.18, S D=7.33)$ y regulación emocional $(M=26.84, S D=7.22)$ que los de centros situados en zonas urbanas (PE: $M=23.48, S D=7.18$;RE: $M=26.34$, $S D=7.23)$, mientras que los individuos de centros situados en el ámbito urbano $(M=24.87, S D=7.21)$ mostraban mayor comprensión emocional que los ubicados en zonas rurales $(M=24.43, S D=7.04)$.

\section{Tabla 5}

Análisis Multivariado de Varianza (MANOVA) del curso escolar, expediente académico, tipo y ubicación del centro en la inteligencia emocional (TMMS-24: subescalas percepción, comprensión y regulación emocional)

\begin{tabular}{lccccc}
\hline \multicolumn{1}{c}{$\begin{array}{c}\text { VARIABLES } \\
\text { INDEPENDIENTES }\end{array}$} & $\begin{array}{c}\text { SUBESCALAS } \\
\text { IE }\end{array}$ & $\boldsymbol{F}$ & $\boldsymbol{p}$ & $\eta_{\mathbf{p}}{ }^{2}$ & Potencia \\
\hline Curso escolar & $\mathrm{PE}$ & 3.43 & .016 & .002 & .78 \\
& $\mathrm{CE}$ & 8.64 & .000 & .006 & .99 \\
& $\mathrm{RE}$ & 29.78 & .000 & .020 & 1 \\
\hline Expediente académico & $\mathrm{PE}$ & 2.45 & .087 & .001 & .49 \\
& $\mathrm{CE}$ & 16.35 & .000 & .007 & 1 \\
& $\mathrm{RE}$ & 20.10 & .000 & .009 & 1 \\
\hline Tipo de centro & $\mathrm{PE}$ & 18.28 & .000 & .004 & .99 \\
& $\mathrm{CE}$ & 2.07 & .150 & .000 & .31 \\
& $\mathrm{RE}$ & 6.64 & .010 & .001 & .73 \\
\hline Ubicación del centro & $\mathrm{PE}$ & 10.45 & .001 & .002 & .90 .58 .67 \\
& $\mathrm{CE}$ & 4.12 & .042 & .001 & \\
\hline
\end{tabular}

Nota . IE = Inteligencia Emocional; PE = Percepción Emocional; CE = Comprensión Emocional; $\mathrm{RE}=$ Regulación Emocional 


\section{DISCUSIÓN Y CONCLUSIONES}

La evidente repercusión de la inteligencia emocional en el ajuste personal, psicológico y social de los adolescentes (Gómez-Baya et al., 2017; Rey et al., 2018; Sánchez-Álvarez et al., 2016), requiere de análisis sistemáticos que den un impulso para su incorporación real al sistema educativo. De ahí que el principal objetivo de esta investigación ha sido analizar de manera exhaustiva, por un lado, el promedio de inteligencia emocional en los adolescentes y, por otro, la influencia de determinadas variables personales y escolares en su percepción, comprensión y regulación emocional.

Por un lado, cabe mencionar que se ha verificado nuestra primera hipótesis planteada $\left(\mathrm{H}_{1}\right.$ : Se infiere un promedio moderado de inteligencia emocional en el periodo adolescente), ya que los datos evidencian una competencia emocional media, en la línea de los trabajos realizados por Cabrera (2011) y Cachay (2017). No obstante, difiere ligeramente del estudio de Pilataxi (2018) que obtiene un nivel medio-bajo en inteligencia emocional del alumnado. Asimismo, los adolescentes de nuestro estudio muestran una mejor regulación emocional que comprensión y, sobre todo, percepción emocional. La investigación realizada por Rey et al. (2011) encontró una mayor claridad y reparación en la inteligencia emocional percibida por los individuos.

De hecho, en este trabajo, los adolescentes centran su inteligencia emocional en prestar atención o preocupación por sus sentimientos, tener un buen estado de ánimo y sentirse felices. Por el contrario, las declaraciones emocionales de menor incidencia se centran en decir cómo se sienten, definir sus sentimientos, tratar de calmarse con cosas complicadas y pensar en los placeres de la vida cuando están tristes. En consecuencia, mejorar la percepción emocional debería ser una prioridad para todas aquellas personas que trabajan con adolescentes, proporcionando líneas educativas más claras a la hora de abordar aspectos emocionales en el proceso de enseñanza-aprendizaje.

Por otro, se ha constatado parcialmente la segunda hipótesis planteada $\left(\mathrm{H}_{2}\right.$. Se predice que las variables personales y escolares presenten diferencias significativas en la percepción, comprensión y regulación emocional durante la adolescencia del individuo), debido a no mostrar diferencias significativas en todas las variables analizadas. Así pues, teniendo en cuenta el género, se revela que las mujeres presentan mayor percepción emocional, mientras que los hombres lo hacen en comprensión y regulación emocional. Estos datos están en la línea de los trabajos de Gartzia et al. (2012), Cazalla-Luna y Molero (2016) o Serrano y Andreu (2016) al señalar que el género ejerce 
un efecto estadísticamente significativo sobre la inteligencia emocional. Por el contrario, estudios como el realizado por Whitman et al. (2009) o Aguilar et al. (2012) no encontraron diferencias significativas entre hombres y mujeres en ninguna de las dimensiones de la inteligencia emocional. No obstante, la mayoría de los estudios muestran que las mujeres tienden a prestar más atención a las emociones que los hombres (Azpiazu et al., 2015; Pulido y Herrera, 2016; Ruiz y Carranza, 2018).

Del mismo modo, en el periodo adolescente se observa que a mayor edad, mejor percepción emocional, mientras que una adolescencia más temprana es indicativa de mejor comprensión y regulación emocional. De hecho, el estudio realizado por Aguilar et al. (2012) señala que a medida que avanza la adolescencia, la habilidad para percibir emociones se hace más importante. Sin embargo, el trabajo realizado por Pulido y Herrera (2018), evidencia un ascenso en las puntuaciones de inteligencia emocional a medida que aumenta la edad.

Con respecto a la estructura familiar, se observa que no es determinante en percepción y comprensión emocional, pero sí en regulación emocional, presentando niveles más óptimos en los adolescentes que viven en familias biparentales. Estos datos son coincidentes con el estudio de Ruiz y Carranza (2018), al indicar que los adolescentes que provenían de familias nucleares presentaban puntuaciones más altas en inteligencia emocional que aquellos que vivían en familias monoparentales.

De manera general, los datos avalan que la inteligencia emocional es mayor en el alumnado con mejores expedientes académicos. A nivel más específico, el alumnado que siempre ha aprobado apunta una mejor comprensión y regulación emocional, siendo significativo indicar que dicha comprensión emocional también es superior en el alumnado repetidor. Dichos resultados están en la línea con estudios anteriores que avalan una fuerte asociación entre el éxito académico y los factores de la inteligencia emocional (Dolev y Leshem, 2017; Costa y Faria, 2015; Gutiérrez et al., 2016). Además, también se presentan diferencias significativas en el curso escolar, con una mejor comprensión y regulación emocional en el primer curso de ESO, mientras la percepción emocional es superior en tercero de ESO.

Atendiendo a la ubicación del centro de enseñanza, se percibe que los núcleos rurales favorecen la percepción y regulación emocional, mientras los urbanos lo hacen en comprensión emocional. Igualmente, el tipo de centro muestra diferencias significativas en la inteligencia emocional de su alumnado, siendo mayor en el alumnado que estudia en centros concertados, sobre todo en percepción y regulación emocional. La investigación 
realizada por Rojas (2017) contradice nuestros datos, al sostener una mejor inteligencia emocional en los estudiantes que viven en la capital frente a los que viven en zonas rurales y mejores resultados en el alumnado de cuarto curso frente a los de tercero, segundo y primero.

En conclusión, el estudio infiere que la inteligencia emocional no está presente en los adolescentes todo lo que debiera, siendo necesario abordarla a través de los factores personales y escolares que rodean al individuo. Como principales limitaciones del estudio indicar que aunque la muestra es muy amplia, serían necesarios estudios de tipo longitudinal que permitieran evaluar el desarrollo de la inteligencia emocional en este periodo adolescente. Además, señalar que los datos se obtuvieron de forma transversal, no pudiendo comprobar la persistencia de los efectos de la inteligencia emocional y las relaciones de causa-efecto en las variables analizadas. Asimismo, otra de las debilidades se encuentra en que la solución factorial alcanzada explica menos de un $60 \%$ de la varianza. También sería recomendable incluir trabajos de investigación sobre esta temática de otros países y culturas.

Como perspectiva de futuro, a pesar de los grandes avances logrados en la medición de la inteligencia emocional, aún queda mucho trabajo por hacer desde los centros educativos, implementando nuevos programas específicos que trabajen sobre estos factores personales y escolares en la escuela, indicando en este sentido la necesidad de abordar el desarrollo de la inteligencia emocional como ayuda para el desempeño social y personal de las personas. Por último, esta investigación contribuye a mejorar el conocimiento de la inteligencia emocional en los adolescentes, cuestión que puede servir a docentes y profesionales para identificar y abordar los déficits emocionales, trabajar su desarrollo y proporcionar líneas educativas más claras a la hora de abordar las emociones. 


\section{REFERENCIAS BIBLIOGRÁFICAS}

Aguilar, C., Calvo, A., \& Monteoliva, A. (2012). La inteligencia emocional percibida y su relación con el apego adulto. Behavioral Psychology/ Psicología Conductual, 20(1), 119-136.

Alonso-Ferres, M., Berrocal de Luna, E., \& Jiménez-Sánchez, M. (2018). Estudio sobre la inteligencia emocional y los factores contextuales en estudiantes de cuarto de educación primaria de la provincia de Granada. Revista de Investigación Educativa, 36(1), 141-158. https://doi.org/10.6018/ rie.36.1.281441

Antonio, I. (2019). Inteligencia Emocional Percibida. Un nuevo instrumento de medida y su contribución a un modelo de ajuste personal y escolar en la adolescencia. [Tesis doctoral. UPV/EHU]. https://dialnet.unirioja.es/ servlet/tesis? codigo $=223660$

Antonio, I., Esnaola, I., \& Rodríguez, A. (2017). La medida de la inteligencia emocional en el ámbito psicoeducativo. Interuniversitaria de Formación del Profesorado, 88(31, 1), 53-64.

Azpiazu, L., Esnaola, I., \& Sarasa, M. (2015). Capacidad predictiva del apoyo social en la inteligencia emocional. European Journal of Education and Psychology, 8, 23-29. https://doi. org/10.1016/j.ejeps.2015.10.003

Bisquerra, R. (coord.) (2012) ¿Cómo educar las emociones? La inteligencia emocional en la infancia y la adolescencia. Hospital Sant Joan de Déu.

Blakemore, S. J., \& Frith, U. (2007). Cómo aprende el cerebro. Las claves para la educación. Ariel.

Cabrera, M. (2011). Inteligencia emocional y rendimiento académico de los alumnos del nivel secundario de una institución educativa de la Región Callao. [Tesis de magíster. Universidad Nacional de San Martín]. http://repositorio.usil.edu.pe/ handle/123456789/1120

Cachay, W.A. (2017). Relación entre el clima social familiar y la inteligencia emocional de los alumnos de quinto grado de nivel secundaria de la I.E.P. "Virgen de la Puerta" Castilla, Piura. [Tesis de Licenciatura. Universidad Católica los Ángeles de Chimbote, Perú]. http://repositorio.uladech.edu. pe/handle/123456789/91

Carrasco, J. D. (2017). Relación entre inteligencia emocional, autoeficacia general y variables sociodemográficas en pacientes ambulatorios del Servicio de Psicología de una clínica de Lima Metropolitana. [Tesis de pregrado. Universidad Peruana Cayetano Heredia]. https://hdl.handle. net/20.500.12866/984

Cazalla-Luna, N., \& Molero, D. (2016). Perceived emotional intelligence, dispositional optimism-pessimism, life satisfaction and teachers' personality in their initial training. Revista de Investigación Educativa, 34(1), 241258. https://dx.doi.org/10.6018/ rie.34.1.220701

Costa, A., \& Faria, L. (2015). The impact of Emotional Intelligence on academic achievement: A longitudinal study in Portuguese secondary school. Learning and Individual Differences, 37, 38-47.

Dolev, N., \& Leshem, S. (2017). Developing emotional intelligence competence among teachers. Teacher Development, 21(1), 21-39. https://doi. org/10.1080/13664530.2016.1207093 
Fernández-Berrocal, P., Extremera, N., \& Ramos, N. (2004). Spanish Modified Version of the Trait Meta-Mood Scale. Psychological Reports, 94, 751-755.

Gartzia, L., Aritzeta, A., Balluerka, N., \& Barberá, E. (2012). Inteligencia emocional y género: más allá de las diferencias sexuales. Anales de Psicología, 28, 567-575. https://revistas. um.es/analesps/article/view/124111

García, M., Hurtado, P., Quintero, D., Rivera, D., \& Ureña, Y. (2018). La gestión de las emociones, una necesidad en el contexto educativo y en la formación profesional. Revista Espacios, 39(49), 8-21.

Gini, G., \& Espelage, D.L. (2014). Peer Victimization, Cyberbullying, and Suicide Risk in Children and Adolescents. JAMA, 312(5), 545-546. https://doi.org/10.1001/ jama.2014.3212

Gómez-Baya, D., Mendoza, R., Paino, S., \& Matos, M.G. (2017). Perceived emotional intelligence as a predictor of depressive symptoms during midadolescence: A two-year longitudinal study on gender differences. Personality and Individual Differences, 104, 303-312. https://doi.org/10.1016/j. paid.2016.08.022

González, A., Peñalver, J., \& Bresó, E. (2011). La evaluación de la inteligencia emocional: ¿Autoinformes o pruebas de habilidad? Forum de Reserca, 16, 699-712.

Gutiérrez, M., Ibáñez, R., Aguilar, R., \& Vidal, A. (2016). Assessment of emotional intelligence in a sample of prospective secondary education teachers. Journal of Education for Teaching, 42, 123-134.https://doi.org/1 0.1080/02607476.2016.1143144

IBM Corp. (2012). SPSS Statistics for Windows, Version 21.0. IBM Corp.
Márquez, C.R. (2016). Relación entre la inteligencia emocional y la motivación laboral del personal de recursos humanos de un ingenio azucarero. [Tesis de Grado. Universidad Rafael Landívar]. http://recursosbiblio.url. edu.gt/tesisjcem/2016/05/43/MarquezCarlos.pdf

Matthews, G., Zeidner, M., \& Roberts, R.D. (2012). Emotional intelligence 101. Springer Publishing Company.

Mavrovelli, S., Petrides, K.V., Rieffe, C., \& Bakker, F. (2007). Trait emotional intelligence, psychological well-being and peer-rated social competence in adolescence. British Journal of Developmental Psychology, 25, 263-275.

Menéndez, D. (2018). Aproximación crítica a la Inteligencia Emocional como discurso dominante en el ámbito educativo. Revista Española de Pedagogía, 269, 07-23. https://doi. org/10.22550/REP76-1-2018-01

Nixon, C.L. (2014). Current perspectives: The impact of cyberbullying on adolescent health. Adolescent Health, Medicine and Therapeutics, 5, 143. https://doi.org/10.2147/AHMT.S36456

Ocaña, J., García, G.A., \& Cruz, O. (2019). Propiedades psicométricas del Trait Meta-MoodScale (TMMS-24) en adolescentes de Chiapas, Mexico. European Scientific Journal, 15(16), 280-294. http://dx.doi.org/10.19044/ esj.2019.v15n16p280

Peña-Casares, M.J., \& Aguaded-Ramírez, E.M. (2019). Evaluación de la inteligencia emocional en el alumnado de educación primaria y educación secundaria. Revista de Educación de la Universidad de Granada, 26, 53-68.

Petrides, K.V., Niven, L., \& Mouskounti, T. (2006). The trait emotional intelligence of ballet dancers and musicians. Psicothema, 18, 101-107. 
Pilataxi, X.E. (2018). Nivel de inteligencia emocional en estudiantes de séptimo y octavo año de Educación General Básica de la Unidad Educativa Diego Abad de Cepeda de la ciudad de Quito en el periodo académico 2016 -2017. Universidad Central de Ecuador. www.dspace.uce.edu.ec/ bitstream/25000/14418/1/T-UCE-0010PEO053-2018.pdf

Ponce, N., \& Aguaded, E.M. (2016). Evaluación de un programa de intervención educativa en inteligencia emocional. Dilemas contemporáneos: Educación, Política y Valores, Año IV(2), art 18. hdl.handle.net/10272/13625

Pulido, F., \& Herrera, F. (2016). Diferencias por género en una validación inicial de un instrumento en construcción para evaluar la inteligencia emocional en una muestra de alumnos de secundaria. Tendencias Pedagógicas, 28, 99-114. http://hdl. handle.net/10486/671829

Pulido, F., \& Herrera, F. (2018). Predictores de la felicidad y la inteligencia emocional en la educación secundaria. Revista Colombiana de Psicología, 27(1), 71-84. https://dx.doi. org/10.15446/rcp.v27n1.62705

Ramos, N., Fernández-Berrocal, P., \& Extremera, N. (2007). Perceived emotional intelligence facilitates Cognitive-emotional processes of adaptation to an acute stressor. Cognition \& Emotion, 21, 758-772.

Rey, L., Extremera, N., \& Pena, M. (2011). Perceived emotional intelligence, self-esteem and life satisfaction in adolescents. Psychosocial Intervention, 20, 227-234. https://doi.org/10.5093/ in2011v20n 2 a 10

Rey, L., Quintana-Orts, C., MéridaLópez, S., \& Extremera, N. (2018). Emotional intelligence and peer cybervictimisation in adolescents: Gender as moderator. Comunicar, 56, 9-18. https://doi.org/10.3916/C56-201801

Rojas, P. (2017). La inteligencia emocional en el contexto educativo de adolescentes cordobeses. Revista de Estudios e Investigación en Psicología y Educación, 5, 5-8.

Ruiz, P., \& Carranza, R. (2018). Inteligencia emocional, género y clima familiar en adolescentes peruanos. Acta Colombiana de Psicología, 21(2), 188-211.https://doi.org/10.14718/ ACP.2018.21.2.9

Salovey, P., \& Mayer, J.D. (1990). Emotional intelligence. Imagination, Cognition and Personality, 9, 185-211.

Sánchez-Álvarez, N., Extremera, N., \& Fernández-Berrocal, P. (2016). The relation between emotional intelligence and subjective well-being: a meta-analytic investigation. The Journal of Positive Psychology, 11(3), 276-285. https://doi.org/10.1080/17439 760.2015.1058968

Saucedo, M., Díaz, J.J., Salinas, H.A., \& Jiménez, S. (2019). Inteligencia emocional; cuestión de género. Revista Boletín Redipe, 8(5), 158-167. https:// revista.redipe.org/index.php/1/article/ view/745

Serrano, C., \& Andreu, Y. (2016). Inteligencia emocional percibida, bienestar subjetivo, estrés percibido, engagement y rendimiento académico en adolescentes. Revista de Psicodidáctica, 21(2), 1-16. http://dx.doi.org./10.1387/ RevPsicodidact.14887.

Taramuel, J.A., \& Zapata, V.H. (2017). Aplicación del test TMMS-24 para el análisis y descripción de la inteligencia emocional considerando la influencia del sexo. Revista Publicando, 4(11,1), 
162-181. https://revistapublicando.org/ revista/index.php/crv/article/view/518

Whitman, D.S., Van Rooy, D.L., Viswesvaran, C., \& Kraus, E. (2009). Testing the second-order factor structure and measurement equivalence of the Wong and Law emotional intelligence scale across gender and ethnicity. Educational and Psychological Measurement, 69(6), 1059-1074. 


\section{PERFIL ACADÉMICO Y PROFESIONAL DE LOS AUTORES}

José Domínguez Alonso. ORCID: https://orcid.org/0000-0002-1844-000X

Profesor Doctor Asociado en la Universidad de Vigo. Director del Centro de Formación y Recursos (Consellería de Educación, Universidades y Formación Profesional). Miembro de la Revista Galega do Ensino e investigador del grupo GIES-10. Sus líneas de investigación prioritarias son: atención a la diversidad, violencia escolar, motivación e inteligencia emocional. E-mail: jdalonso@uvigo.es

Begoña Nieto Campos. ORCID: https://orcid.org/0000-0002-4232-2089

Doctora por la Universidad de Vigo. Asesora del Centro de Formación y Recursos (Consellería de Educación, Universidades y Formación Profesional). Sus líneas de investigación prioritarias son: violencia escolar e inteligencia emocional. E-mail: bnieto@edu.xunta.es

Iago Portela Pino. ORCID: https://orcid.org/0000-0002-4232-2089

Profesor Doctor Investigador en la Universidad Isabel I (Burgos). Investigador del grupo GIES-10. Sus líneas de investigación están centradas en el análisis de motivaciones, barreras y nivel de actividad física en diferentes poblaciones y su relación. E-mail: iagoportt92@ gmail.com

Fecha Recepción del Artículo: 25. Marzo. 2021

Fecha Modificación del Artículo: 29. Mayo. 2021

Fecha Aceptación del Artículo: 30. Mayo. 2021

Fecha Revisión para Publicación: 13. Septiembre. 2021 